\title{
Nonlinear elastic buckling and postbuckling analysis of cylindrical panels
}

\author{
Yang Zhou ${ }^{\mathrm{a}}$, Ilinca Stanciulescu ${ }^{\mathrm{a}, *}$, Thomas Eason ${ }^{\mathrm{b}}$, Michael Spottswood ${ }^{\mathrm{b}}$ \\ ${ }^{a}$ Rice University, Department of Civil and Environmental Engineering, 6100 Main \\ Street, Houston, TX, 77005, U.S.A \\ ${ }^{b}$ Air Force Research Laboratory, Structural Sciences Center, 2790 D. Street, WPAFB, \\ OH, U.S.A.
}

\section{Abstract}

This paper revisits the buckling analysis of a benchmark cylindrical panel undergoing snap-through when subjected to transverse loads. We show that previous studies either overestimated the buckling load and identified a false buckling mode, or failed to identify all secondary solution branches. Here, a numerical procedure composed of the arclength and branch switching methods is used to identify the full postbuckling response of the panel. Additional bifurcation points and corresponding secondary paths are discovered. Parametric studies of the effect of the rise and thickness of the panel on the buckling and postbuckling responses are also performed.

Keywords: Nonlinear buckling and postbuckling analysis, the arclength method, the branch-switching method, critical points, secondary paths

\footnotetext{
*Corresponding author. Tel.: +1713 348 4704; fax: +1713 3485268 .

Email address: ilinca.s@rice.edu (Ilinca Stanciulescu)
} 


\section{Introduction}

Cylindrical shells are widely used in aerospace, mechanical, and civil engineering applications as structural components in aircraft, tanks, pipelines, and offshore platforms. These structures have efficient load-carrying capabilities but exhibit high risk of buckling failures.

Early studies on the buckling analysis of cylindrical shells used the classical buckling theory to approximate the bucking loads and mode shapes by assuming membrane prebuckling stress states [1 4]. This approach ignores bending effects before buckling and usually overestimates buckling loads. Later, more rigorous buckling analyses were performed with the consideration of linear prebuckling deformations [5] 8] and nonlinear prebuckling deformations [9, 10], but did not focus on postbuckling responses. Koiter [11] proposed a perturbation approach to conduct initial postbuckling analysis, which was later adopted by many researchers [12 15]. These methods are typically valid only in the vicinity of critical points. Potier-Ferry and coworkers [16 19] extended Koiter's idea and developed an asymptoticnumerical method to compute nonlinear postbuckling responses.

Other numerical approaches widely used to perform nonlinear postbuck- 
ling analysis of shell structures are path following schemes. Among them, the Newton-Raphson methods were initially attractive for solving large nonlinear systems but they usually lose convergence at limit points and cannot trace the unstable equilibrium paths. Some of these disadvantages were solved by replacing the load control with displacement control [20, 21], but this approach still fails to track the whole postbuckling path beyond a displacement limit point. Riks [22] proposed a more efficient arclength method that can trace the entire (stable and unstable) postbuckling equilibrium paths. Modified versions were later proposed by Crisfield [23] and Tsai et al. [24] to handle more complicated postbuckling behavior.

Despite the great progress made in the path following approaches, some features of the postbuckling behavior still remained unnoticed. A circular cylindrical panel, studied by Sabir [25], was afterwards used by many researchers [26-35] as a benchmark example to demonstrate the capability of shell or shell-like elements in simulating large deformations buckling and postbuckling processes. All these researchers successfully identified the limitpoint buckling and the corresponding symmetric postbuckling responses by utilizing path following methods. However, these studies did not correctly identify the physical buckling behavior of this panel. Recently, Wardle et 
al. [36, 37] found using the asymmetric meshing technique (AMT) that a bifurcation buckling in asymmetric mode exists before the first limit point on the equilibrium path.

In this work, an arclength method combined with a branch-switching method [38, 39] is used to perform the nonlinear buckling and postbuckling analysis of cylindrical panels. For the benchmark example, we find that two previously undetected pairs of bifurcation points and consequently two other pairs of secondary paths exist. A small interval of one secondary path is stable, while the other equilibria on this path and all states on the other path are unstable. Intervals of stable equilibria identified on secondary paths, while not reachable through a continuous stable path, are still important: perturbations in the system may lead to dynamic jumps to these states. The identification of additional unstable equilibria also reveals that the degree of instability of the system is higher than what researchers previously found.

The numerical approach used in this paper has several advantages over the AMT recommended by Wardle et al. [36, 37]: (1) no prior knowledge of the bifurcation modes is needed, and (2) the same mesh is used for tracing all secondary paths of the structure. The accuracy and reliability of this method is tested on the benchmark example. 
This paper is organized as follows. In Section 2, we briefly introduce the nonlinear buckling analysis algorithm. In Section 3, we apply the method to a benchmark example and compare with the results available in the literature. Additional bifurcation points and secondary paths are also obtained in this section. In Section 4, we perform a parametric study of the influence of the rises, thicknesses and boundary conditions on the variation of critical points and postbuckling responses. Conclusions are outlined in Section 5.

\section{Nonlinear buckling and postbuckling analysis}

In this section, we briefly introduce a numerical procedure, combining the arclength and branch-switching methods, which can reliably determine all critical points and corresponding postbuckling responses including bifurcated secondary paths.

\subsection{Critical points on the equilibrium path}

An elastic system typically loses stability when the tangent stiffness $\mathbf{K}$ becomes singular. Points on the equilibrium path with singular tangent stiffness are called critical points, further differentiated as limit and bifurcation

points (Fig. 1). A null right eigenvector $\mathbf{z}$ of the tangent stiffness $\mathbf{K}$ at a 
critical point satisfies:

$$
\mathbf{K} \mathbf{z}=\mathbf{0}
$$

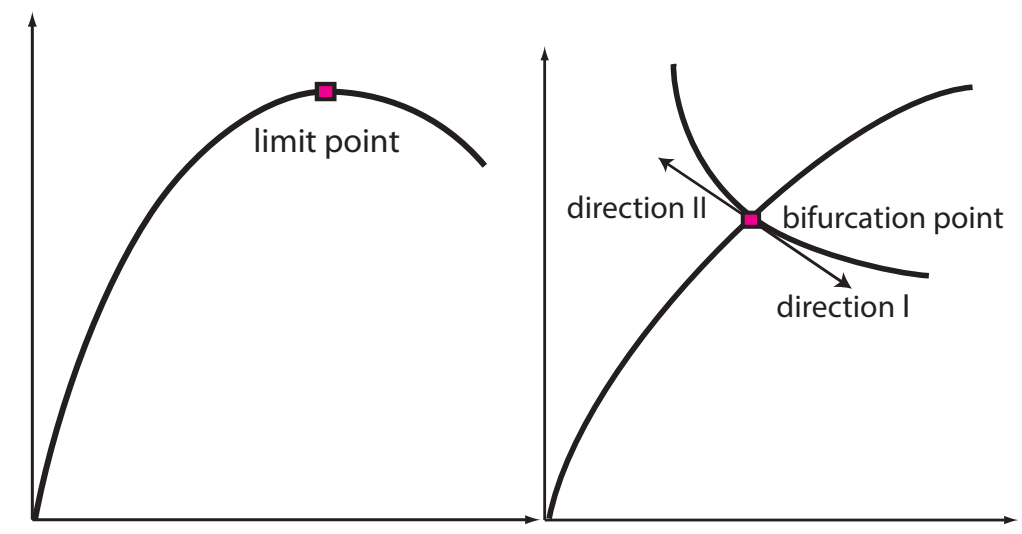

(a) A limit point

(b) A bifurcation point

Figure 1: Critical points on equilibrium paths.

When an elastic structure is subjected to a conservative loading, the tangent stiffness $\mathbf{K}$ is symmetric and Eq. (1) also implies $\mathbf{z}^{T} \mathbf{K}=\mathbf{0}$. For an incremental-iterative method, the incremental displacement $\Delta \mathbf{u}$ and loading $\Delta \lambda$ satisfy $\mathbf{K} \Delta \mathbf{u}=\Delta \lambda \mathbf{q}$. Premultiplying both sides of with $\mathbf{z}^{T}$ and using $\mathbf{z}^{T} \mathbf{K}=\mathbf{0}$, we get

$$
\mathbf{z}^{T} \mathbf{q} \Delta \lambda=0
$$

Three configurations satisfy Eq. (2): (1) $\Delta \lambda=0$, denoting a limit point (Fig. 1a); (2) $\mathbf{z}^{T} \mathbf{q}=0$, indicating a bifurcation point (Fig. 1b); or (3) $\Delta \lambda=0$ and $\mathbf{z}^{T} \mathbf{q}=0$ simultaneously, implying the coincidence of a bifurcation and 
limit point. In practice, limit points are indeed identified as points of zero variation in the load factor, but bifurcation points are not detected based on the above. Instead in this paper, several lowest eigenvalues of the tangent stiffness $\mathbf{K}$ are monitored when tracing the primary equilibrium path. Zero eigenvalues of the tangent stiffness indicate the location of critical points, out of which, those not already identified by $\Delta \lambda=0$ are the bifurcation points. For the case of a multiple bifurcation point or of the coincidence of a limit point and a bifurcation point, multiple eigenvalues are zero at the same time. Finally, note that only conservative systems are considered in this paper.

\subsection{Switching to secondary paths}

After the detection of bifurcation points, the branch-switching method proposed in [38, 39] is adopted to switch from the primary equilibrium path to a secondary path. At a simple bifurcation point, the eigenvector $\phi_{j}$ of the zero eigenvalue $\lambda_{j}$ indicates the direction of one secondary path $\mathbf{j}$, and can be used as a perturbation of the solution on the primary path. To switch to the secondary path $\mathbf{j}$, the eigenvector $\phi_{j}$ is scaled and added to the solution in the following way:

$$
\mathbf{u}_{j}=\mathbf{u} \pm \frac{\|\mathbf{u}\|}{\tau_{j}} \frac{\phi_{j}}{\left\|\phi_{j}\right\|}
$$


where, $\tau_{j}$ is a scaling factor, $\mathbf{u}$ is the converged displacement vector on the primary path, and $\mathbf{u}_{j}$ represents the a predictor for the secondary path $\mathbf{j}$. The arclength method can then be used to correct the predictor $\mathbf{u}_{j}$ and follow additional solutions on the secondary path $\mathbf{j}$.

Two important aspects of this branch-switching method are noted here. First, two directions are typically associated with one secondary path, as shown in Fig. $1 \mathrm{~b}$ and they correspond to the plus and minus sign in Eq. (3). Second, the value of the scaling factor $\tau_{j}$ is usually less than 100 based on our simulation experience (a too large value can lead to a solution that remains on the primary path, while a too small one may lead to divergence). An adaptive approach with a restart option that can rerun a new simulation directly from the bifurcation point is therefore recommended to alleviate the computational cost of possible adjustment of $\tau_{j}$, as used in this paper.

The branch-switching method can also deal with a multiple bifurcation point, where multiple eigenvalues become zero simultaneously. The eigenvectors of the zero eigenvalues at the multiple bifurcation point span a space that contains the directions of possible secondary paths. In general, two kinds of bifurcation buckling are possible: single-mode and multiple-mode buckling. For the single-mode buckling case, the Eq. (3) can be used to determine the 
corresponding secondary path, while for the multiple-mode buckling case, a linear combination of all related eigenvectors can be used to predict the secondary path:

$$
\mathbf{u}_{j}=\mathbf{u} \pm \sum_{i=1}^{n} \frac{\|\mathbf{u}\|}{\tau_{i}} \frac{\phi_{i}}{\left\|\phi_{i}\right\|}
$$

where, $\mathbf{u}_{\mathbf{j}}$ is a predictor on the multiple-mode bifurcated path. The arclength method can then be used to correct the predictor and trace the remaining solutions on this secondary path.

\subsection{The combined numerical procedure}

The combined numerical procedure used to perform a thorough nonlinear buckling and postbuckling analysis of cylindrical panels is summarized as follows: (1) the primary equilibrium path is traced by the arc-length method while monitoring the lowest eigenvalues $\lambda_{j}$ of the tangent stiffness $\mathbf{K}$; (2) all critical points on the primary path are found by identifying all zero eigenvalues $\lambda_{j} ;(3)$ the critical points are classified into limit and bifurcation points by checking whether $\Delta \lambda=0 ;(4)$ the bifurcation points are differentiated into simple and multiple bifurcation points by the multiplicity of zero eigenvalues;

(5) the branch-switching method (Eq. (3) or (4)) is used to switch from the 
primary path to a secondary path at a bifurcation point; (6) the remaining solutions on the secondary path are traced using the arclength method; and (7) If another secondary path is present, a restart option is used to go directly to the bifurcation point and repeat steps (4) and (5).

\section{Benchmark example and solutions}

The benchmark example is a circular cylindrical panel (radius $\mathrm{R}=2540 \mathrm{~mm}$ and thickness $\mathrm{t}=6.35 \mathrm{~mm}$ ) with simply supported longitudinal edges of length $\mathrm{a}=508 \mathrm{~mm}$ and free curved circumferential edges of projected length $\mathrm{b}=507.15 \mathrm{~mm}$ (Fig. 2). The material is isotropic with Young's modulus $\mathrm{E}=3102.75 \mathrm{MPa}$, and Poisson's ratio $\nu=0.3 . \mathrm{P}_{C}$ represents the symmetry plane perpendicular to the circumferential edge and $\mathrm{P}_{L}$ is the symmetry plane perpendicular to the longitudinal edge. A point load is applied in positive $\mathrm{z}$ direction at the center of the panel. The numerical simulations are performed with the Finite Element Analysis Program (FEAP), a research code that includes most commonly used finite element algorithms and provides a reliable framework for developing new user formulations [40]. 


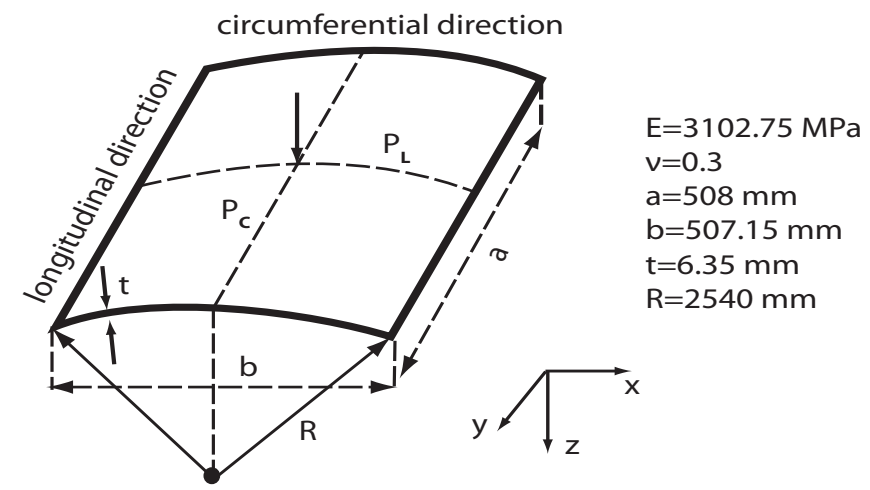

Figure 2: Benchmark cylindrical panel.

\subsection{Limit points and primary equilibrium path}

Due to the symmetric geometry, loading and boundary conditions of the panel, many researchers 25 35] intuitively assumed that this problem only has symmetric solutions and performed the analysis on one quarter of the panel to save computational time. Such approach completely eliminates the possibility of identifying possible bifurcation points and secondary paths.

In this paper, the full panel is modeled with 27-node solid elements, which are free of locking. Alternative ways to avoid locking are also available using an 8-node solid element or a shell element, with enhanced strain formulation or reduced integration. Fig. 3 shows a mesh convergence study for the 27 node solid element, where the number of elements in the circumferential and longitudinal directions are increased, while one element is always used in the thickness direction. The limit load obtained using the most refined 
mesh (2304 elements) is used as a reference value for calculating the relative errors in Fig. 3b. All the following simulations are performed using 144 27node solid elements that lead to less than $1.8 \%$ relative error. The simply supported boundary condition is enforced using the nodes in the middle layer of each longitudinal edge.

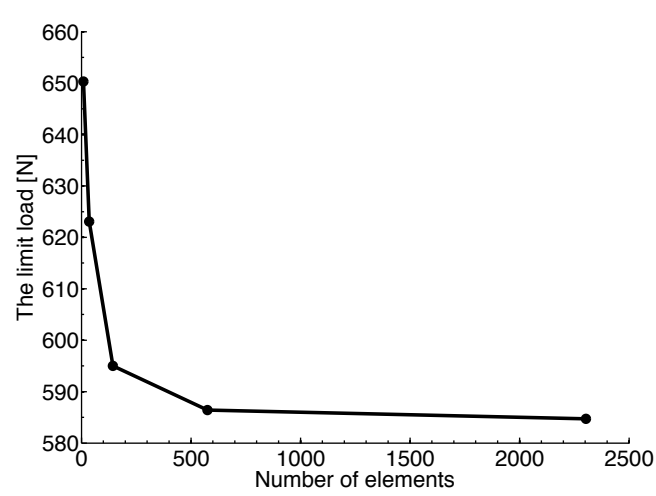

(a) The limit load

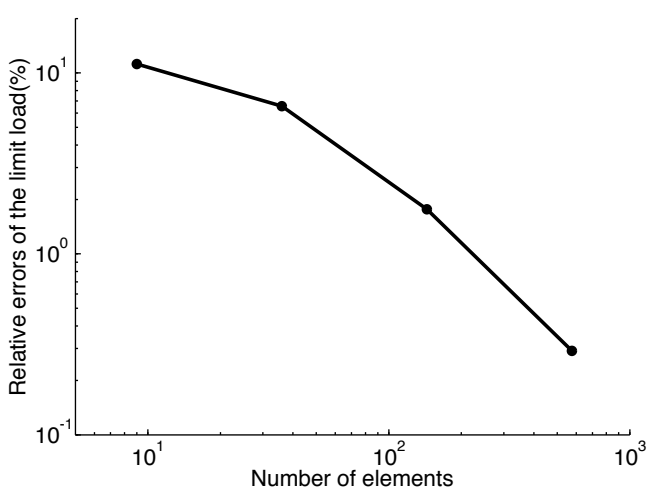

(b) The relative errors

Figure 3: A mesh convergence study

An arclength method is used to trace the primary path (Fig. 4a) and the obtained solution (solid line) is compared to the result from [35] (squares). The central deflection is the positive z-direction displacement of the center point of the panel (Fig. 22). On the equilibrium path, one pair of load-limit points (points with horizontal tangents) can be identified, indicating that the panel exhibits limit-point buckling at $\mathrm{P}_{l i m}=595.20 \mathrm{~N}$. Fig. $4 \mathrm{~b}$ shows the associated buckling mode that is symmetric to both center planes $\mathrm{P}_{C}$ and 
$\mathrm{P}_{L}$ (Fig. 2).

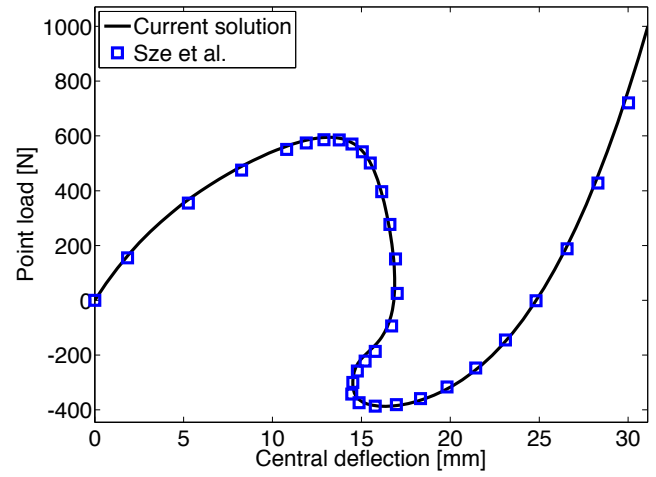

(a) Primary equilibrium path

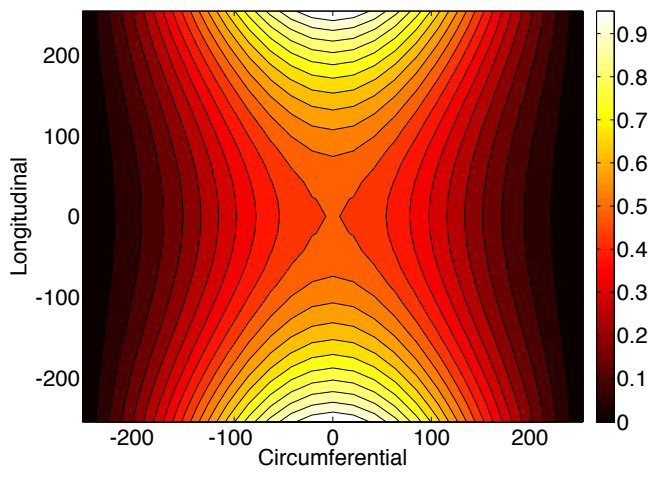

(b) Limit-point buckling mode

Figure 4: Symmetric solutions of the benchmark example

\subsection{Secondary equilibrium paths}

To identify bifurcation points, several lowest eigenvalues of the tangent stiffness of the system are monitored when tracing the primary equilibrium path. A branch-switching method [38, 39] is then employed to switch to secondary paths at bifurcation points.

Fig. 5a shows the two lowest eigenvalues of the tangent stiffness on the primary equilibrium path: they are initially positive at zero load, and decrease as the applied load increases. This plot identifies a bifurcation point at the location where the lowest eigenvalue becomes zero (at a load smaller than the limit load). At this bifurcation point, the branch-switching method is adopted to switch to the secondary path. Fig. $5 \mathrm{~b}$ shows this secondary 

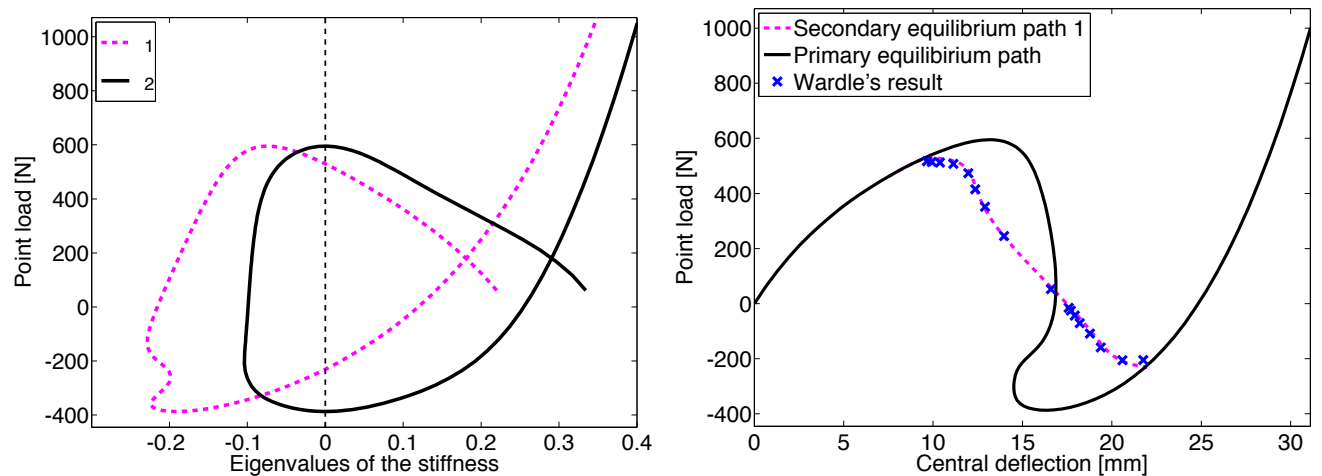

(a) Two lowest eigenvalues of the tangent(b) Primary and first secondary equilibrium stiffness on the primary path path

Figure 5: Previously known solution paths of the benchmark example.

equilibrium path (dashed line) together with the primary equilibrium path (solid line). The secondary equilibrium path obtained in [36] using the AMT is also shown in this figure for comparison (cross markers). The AMT requires modeling the structure with an asymmetric mesh that has the same pattern as the corresponding bifurcation mode, typically not available but "assumed" when generating the mesh, which can lead to incorrect results.

Here, the bifurcation buckling load is $537.10 \mathrm{~N}$, which identifies the critical load at $90 \%$ of the limit load (595.20 N), the overestimated critical value predicted by [25]35. Fig. 6a shows the bifurcation buckling mode, which is asymmetric to the center plane $\mathrm{P}_{C}$, and symmetric to the center plane $\mathrm{P}_{L}$. The stability of this secondary equilibrium path can be determined by mon- 


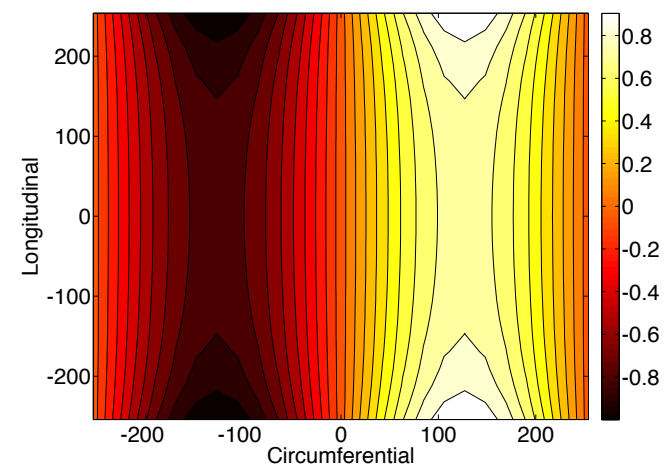

(a) Bifurcation buckling mode

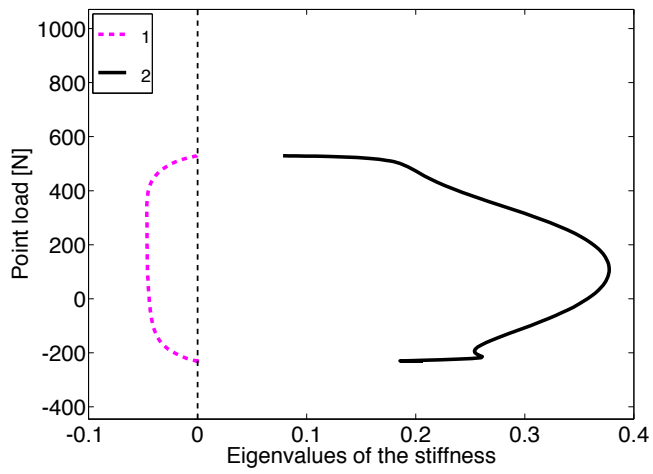

(b) Two lowest eigenvalues

Figure 6: Characteristics of the secondary equilibrium path 1

itoring the eigenvalues of the tangent stiffness on the secondary equilibrium path (Fig. 6b). It can be observed from this figure that the lowest eigenvalue $\lambda_{1}$ is always negative. Thus, the entire secondary equilibrium path 1 is unstable.

\subsection{Additional secondary equilibrium paths}

Although Wardle et al. [36, 37] successfully obtained one secondary equilibrium path for the benchmark example, they did not recognize that multiple pairs of bifurcation points and secondary equilibrium paths exist. In this subsection, two other pairs of bifurcation points and secondary equilibrium paths are identified. Note that this analysis is still performed on the original mesh, unlike the AMT that would require two more meshes for this task.

In order to identify all critical (limit and bifurcation) points, more eigen- 


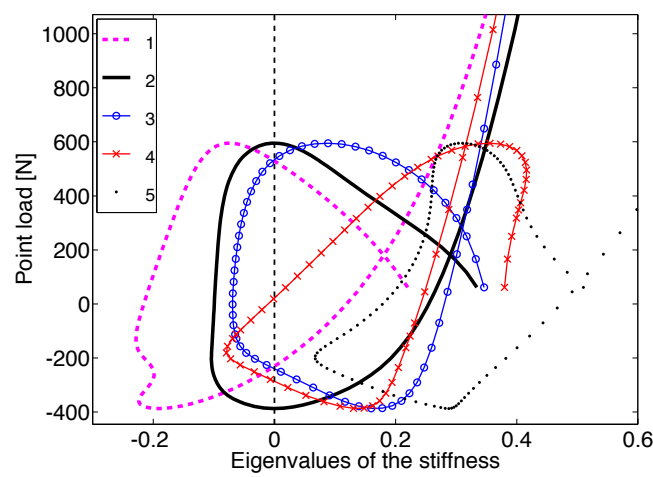

(a) Five lowest eigenvalues

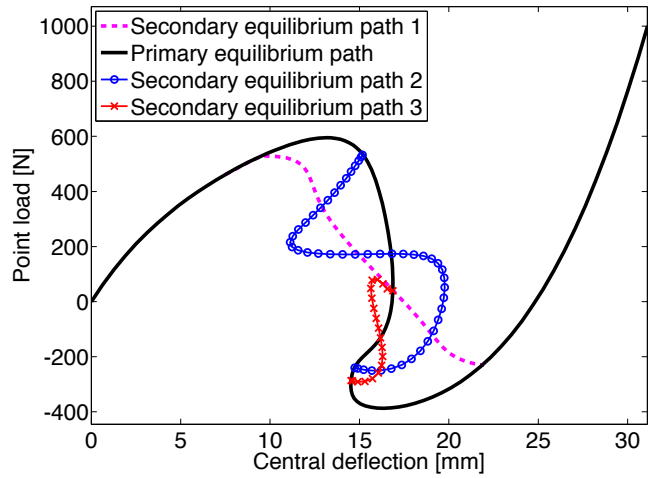

(b) All connected equilibrium paths

Figure 7: Solutions of the benchmark example.

values of the tangent stiffness are monitored. Fig. 7 a shows the five lowest eigenvalues of the tangent stiffness on the primary equilibrium path. It can be seen from this figure that the eigenvalue of the fifth mode (dotted line) is the lowest that is always positive: the third and fourth eigenvalues become negative indicating additional bifurcations. Two more pairs of bifurcation points are thus identified and the corresponding secondary equilibrium paths are obtained. The primary equilibrium path and all three secondary equilibrium paths are shown in Fig. $7 \mathrm{~b}$. The bifurcation buckling mode of the secondary equilibrium path 2 (Fig. 8a), is asymmetric with respect to the center plane $\mathrm{P}_{L}$ but symmetric with respect to the center plane $\mathrm{P}_{C}$. The secondary equilibrium path 3 is associated with the bifurcation buckling mode that is asymmetric with respect to both center planes $\mathrm{P}_{C}$ and $\mathrm{P}_{L}$, but sym- 
metric with respect to the center point of the panel (Fig. 8b).

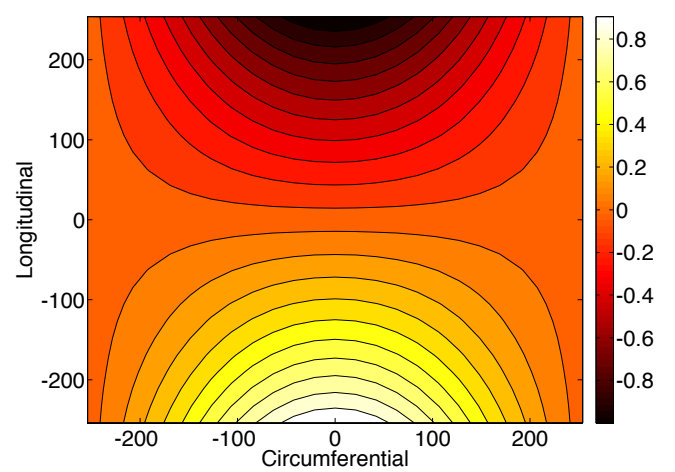

(a) Secondary equilibrium path 2

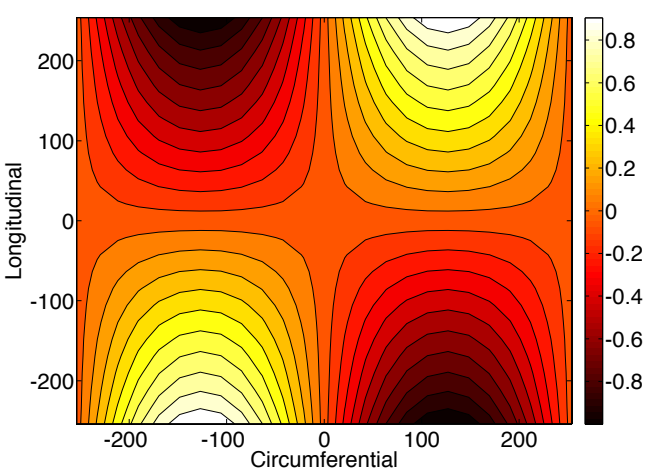

(b) Secondary equilibrium path 3 .

Figure 8: Additional bifurcation buckling modes

To reveal the stability of secondary equilibrium paths 2 and 3, eigenvalues of the tangent stiffness on these secondary equilibrium paths are examined. Fig. 9a shows the three lowest eigenvalues of the tangent stiffness on the secondary equilibrium path 2 . It can be seen that all eigenvalues are positive for the load interval $171.6 \mathrm{~N}$ to $173.8 \mathrm{~N}$, indicating that the equilibrium states in this loading range, represented by solid line in Fig. 9b, are stable on the secondary equilibrium path 2. Fig. 10 shows the four lowest eigenvalues of the tangent stiffness on secondary equilibrium path 3. Two eigenvalues are always negative for all the states on this path, denoting that the entire secondary path 3 corresponds to unstable states.

Snap-through is dynamic process and a full understanding of the post 


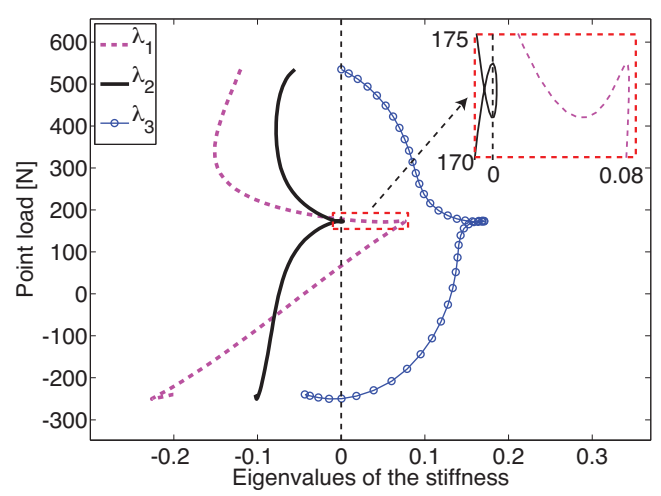

(a) Three lowest eigenvalues

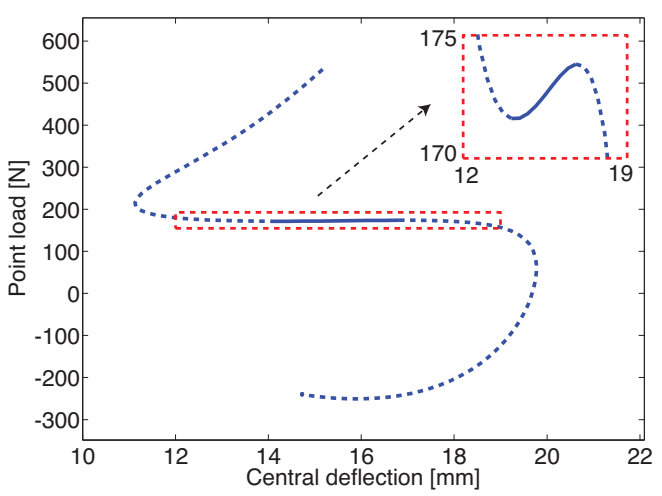

(b) Equilibrium path

Figure 9: Stability of equilibria on secondary path 2

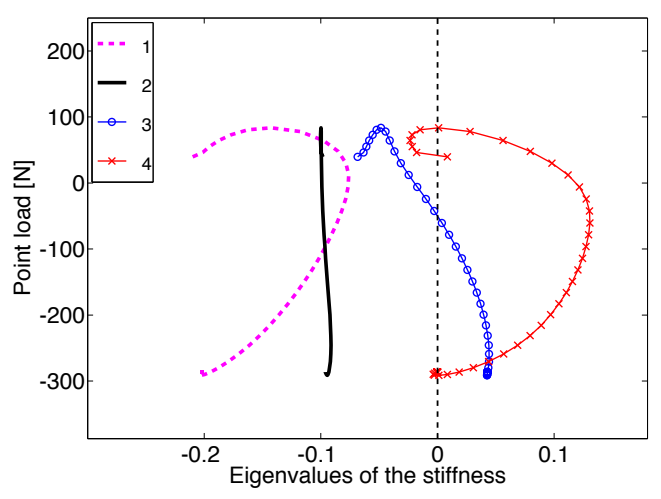

Figure 10: The four lowest eigenvalues of the tangent on secondary path 3.

snap response can only be obtained through a transient analysis. However, the identification of additional pairs of bifurcation points and secondary paths leads to a more thorough understanding of the buckling and postbuckling behavior of the panel. Moreover, all these equilibria, stable or unstable, provide useful insight into the dynamic characteristics of the system. First, the stable equilibria on the secondary path 2 could potentially be reached under 
certain conditions (perturbations). Second, unstable equilibria are potential energy hilltops and act as repulsors in the dynamic behavior of the system. The energy hilltops and ridges between them serve as approximate basins of attractions for the two stable equilibria. Third, the presence of additional unstable branches indicates that the benchmark example has a degree of in-

stability higher than what researchers previously found. Knowledge of the degree of instability and corresponding modes provide valuable information in choosing adequate algorithms for the transient analysis and avoiding the inconsistent stability of time integrators that plagues the simulation of systems with risk of snap-through[41].

\section{Parametric studies of the cylindrical panel}

The nonlinear buckling behavior of shell structures is greatly influenced by the geometry and boundary conditions. Here, an investigation of the influence of the rise, thickness and boundary conditions on the nonlinear buckling responses is conducted. In all following figures, secondary equilibrium paths are classified into three categories based on the types of asymmetric deformation modes obtained. We denote as path $\mathrm{C}$ an equilibrium path asymmetric to center plane $\mathrm{P}_{C}$ but symmetric to center plane $\mathrm{P}_{L}$ (Fig. 6a), 
as path $\mathrm{L}$ an equilibrium path asymmetric to center plane $\mathrm{P}_{L}$ but symmetric to center plane $\mathrm{P}_{C}$ (Fig. 8a), and as path $\mathrm{B}$ an equilibrium path asymmetric to both planes (Fig. 8b), but symmetric to the center point of the panel.

\subsection{The influence of the rise on the buckling behavior}

The rise of the cylindrical panel in the benchmark example is $\mathrm{h}=12.68 \mathrm{~mm}$. Here, we conduct a parametric study by varying the rise from $3.04 \mathrm{~mm}$ to $14.32 \mathrm{~mm}$. No critical point exists and consequently buckling does not occur for panels with small rise (Fig. 11a). The structure displays an initial softening behavior followed by stiffening. When the rise of the panel is increased, the structure exhibits limit-point buckling with one pair of load-limit points (Fig. 11b). As the rise of the panel is further increased, a secondary path L appears after the first limit point (Fig. 11c). The structure with this rise loses stability when the first limit point is reached.

Figs. 11d, 11e and 11f show equilibria of cylindrical panels with higher rises that have two types of secondary paths. The new secondary path $\mathrm{C}$ initially appears between the bifurcation points of secondary path L, as shown in Fig. 11d, When the rise of the panel is further increased, these secondary paths become longer and the bifurcation points move toward the limit points. In Fig. 11f, the secondary path $\mathrm{C}$ appears earlier than secondary path $\mathrm{L}$. 


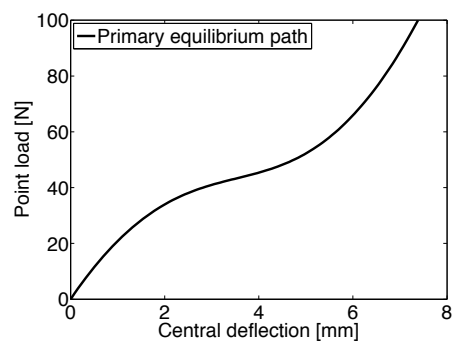

(a) $\mathrm{h}=3.04 \mathrm{~mm}$

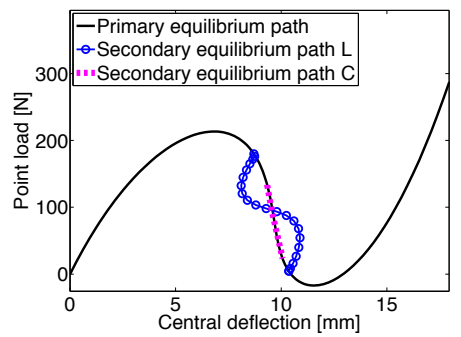

(d) $\mathrm{h}=7.73 \mathrm{~mm}$

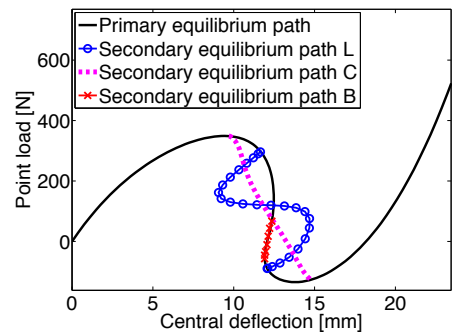

(g) $\mathrm{h}=9.76 \mathrm{~mm}$

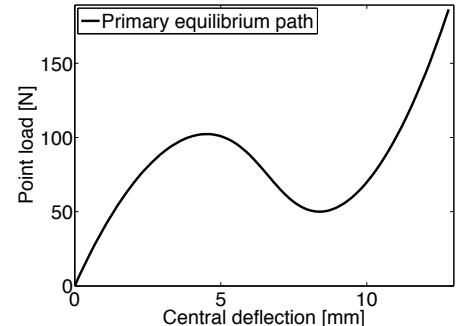

(b) $\mathrm{h}=5.45 \mathrm{~mm}$

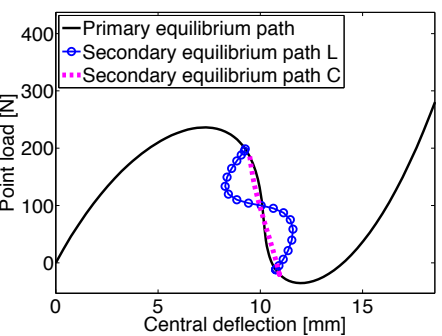

(e) $\mathrm{h}=8.11 \mathrm{~mm}$

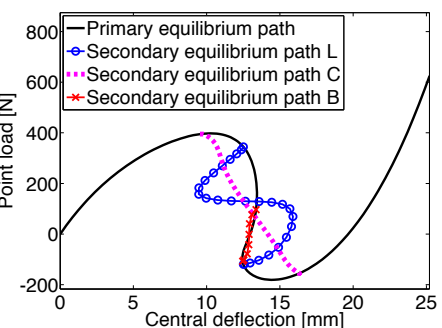

(h) $\mathrm{h}=10.40 \mathrm{~mm}$

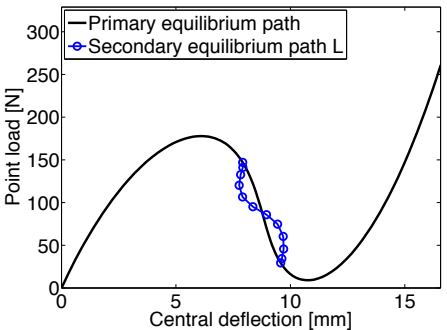

(c) $\mathrm{h}=7.10 \mathrm{~mm}$

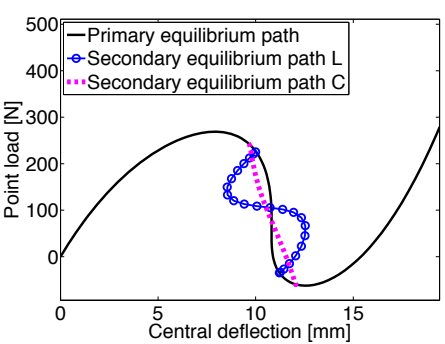

(f) $\mathrm{h}=8.62 \mathrm{~mm}$

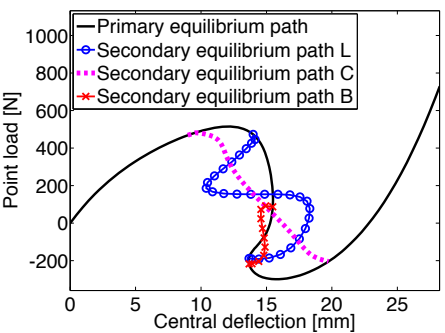

(i) $\mathrm{h}=11.79 \mathrm{~mm}$

Figure 11: Equilibrium paths of panels with different rises.

Under static load, panels with these rises still buckle symmetrically at the

first limit point. A panel with higher rises has three pairs of secondary paths (Fig. 11g, 11h and 11i). The last secondary path B initially appears between the bifurcation points of secondary path L (Fig. 11g). As the rise is increased, the secondary path C starts before the first limit point (Fig. 11h). 
Therefore, a panel with this rise bifurcates asymmetrically before reaching the limit point. When the rise of the panel is further increased, the ratio of the critical load identified at the bifurcation point of the secondary path $\mathrm{C}$ to the load of the first limit point continues to decrease, but secondary paths $\mathrm{L}$ and B still occur after the first limit point.

\subsection{The influence of the thickness on the buckling behavior}

The thickness of the benchmark cylindrical panel is $\mathrm{t}=6.35 \mathrm{~mm}$. The following parameter study examines the influence of thickness, which is varied from $22.86 \mathrm{~mm}$ to $5.59 \mathrm{~mm}$ while keeping all other parameters unchanged. Fig. 12 shows the variation of all equilibrium paths when changing the thickness of the panel, which identifies the same trend as shown in Fig. 11 and indicates that decreasing the thickness of the panel has a similar influence on the number and location of critical points and the shapes of postbuckling responses as increasing the rise of the panel.

It can be concluded that the number and location of critical points and the shapes of postbuckling responses are sensitive to the variation of the rise and thickness of a cylindrical panel. Multiple pairs of bifurcation points and secondary paths exist for many cases discussed above. Thus, a robust numerical procedure, like the one recommended in this paper, which can reliably 


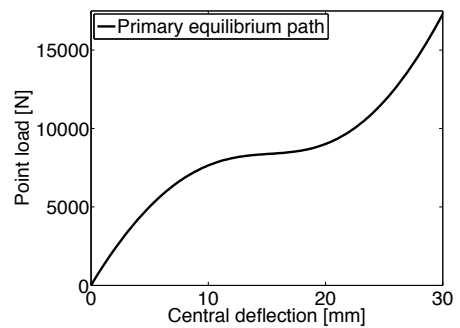

(a) $\mathrm{t}=22.86 \mathrm{~mm}$

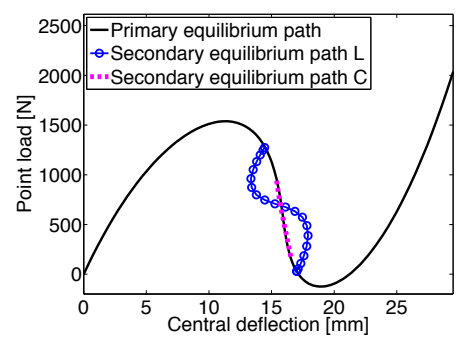

(d) $\mathrm{t}=10.41 \mathrm{~mm}$

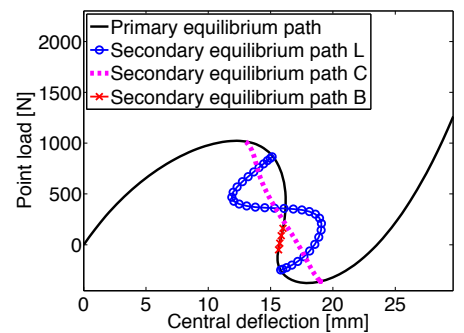

(g) $\mathrm{t}=8.38 \mathrm{~mm}$

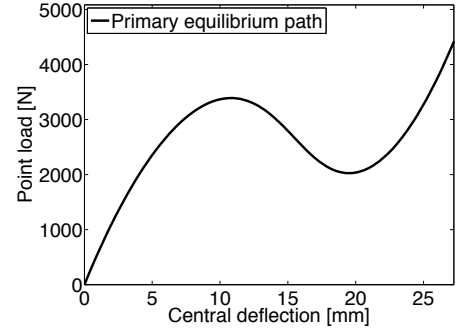

(b) $\mathrm{t}=15.75 \mathrm{~mm}$

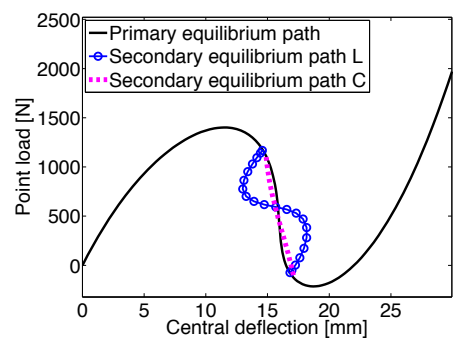

(e) $\mathrm{t}=9.14 \mathrm{~mm}$

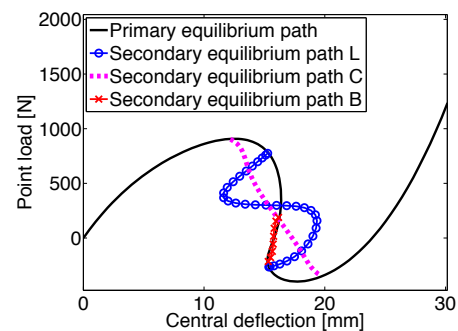

(h) $\mathrm{t}=7.87 \mathrm{~mm}$

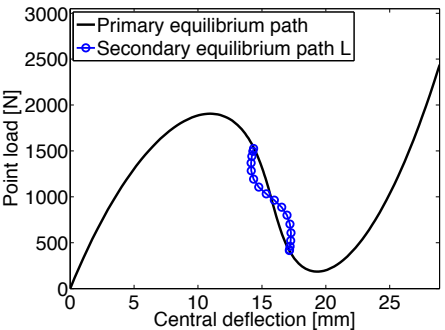

(c) $\mathrm{t}=11.68 \mathrm{~mm}$

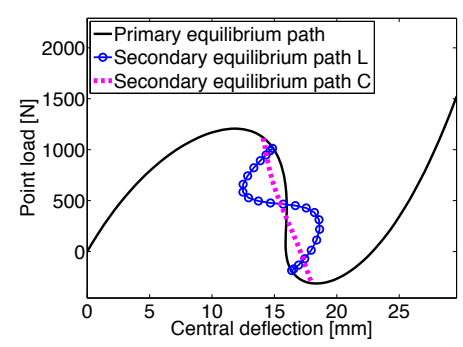

(f) $\mathrm{t}=9.90 \mathrm{~mm}$

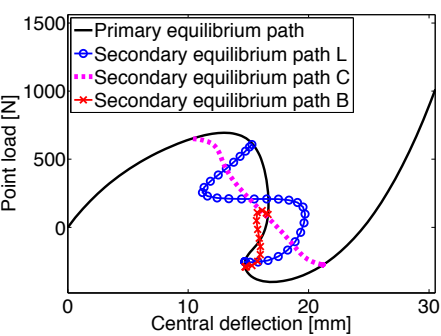

(i) $\mathrm{t}=6.86 \mathrm{~mm}$

Figure 12: Equilibrium paths of panel with different thickness.

detect all critical points and obtain corresponding postbuckling responses in-

cluding all secondary paths is necessary for performing a thorough buckling and postbuckling analysis of cylindrical panels. If only the arclength method is used, all bifurcation points and secondary paths in Fig. $11 \mathrm{c}$ to $11 \mathrm{i}$ and Fig. 12c to $12 \mathrm{i}$ can not be detected. Secondary paths L and B in these plots 
still can not be obtained even when the AMT with the specifically assumed bifurcation mode recommended in [36] is employed.

\subsection{The influence of boundary conditions on the buckling behavior}

This study uses the same geometry and external load of the benchmark example, while the boundary conditions are changed. When a 3D solid element is used to model the cylindrical panel, multiple layers of nodes exist in the thickness direction. The choice of the layer of nodes to be constrained greatly influences the buckling and postbuckling responses.

Fig. 13 shows the primary equilibrium paths obtained by applying the same constraint (restricting translations in $\mathrm{x}, \mathrm{y}$, and $\mathrm{z}$ direction) to the nodes on three different layers. The constraint on the top layer leads to the lowest limit load (black dashed line), while the support on the bottom layer (red dotted line) results in the largest limit load. The postbuckling responses on the primary path are also very different; two displacement-limit points exist for the constraint on the middle layer.

The number and location of bifurcation points and secondary paths are also very sensitive to the boundary constraint. Fig. 14 shows the primary and secondary paths when the constraint is applied to nodes on the top and bottom layers, while the results of the constraint on the middle layer (three 


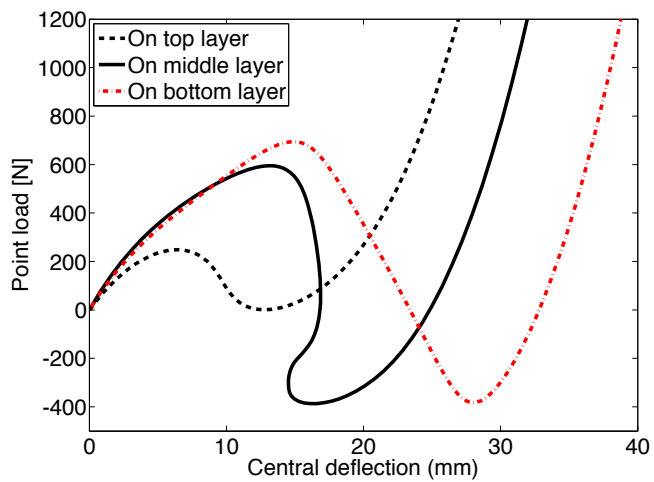

Figure 13: Primary equilibrium paths of supports on three different layers

pairs of bifurcation points and secondary paths) are already shown in Fig. $7 \mathrm{~b}$. The secondary path $\mathrm{C}$ appears before the first limit point, while secondary paths L and B appear after the limit point. In contrast, only one pair of bifurcation points and a secondary path $\mathrm{L}$ exist after the first limit point for the constraint on the top layer (Fig. 14a), and only two pairs of bifurcation points, and secondary paths $\mathrm{C}$ and $\mathrm{L}$ appear after the first limit point when the constraint is applied to the bottom layer (Fig. 14b).

\section{Concluding remarks}

This paper presents a finite element buckling and postbuckling analysis of cylindrical panels. It is shown that many previous studies of a commonly used benchmark cylindrical panel [25]35] overestimated the buckling load and failed to identify the correct buckling mode shape. A numerical pro- 


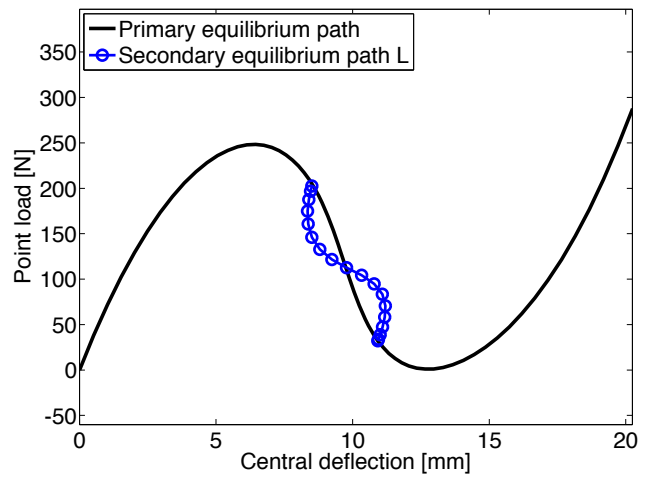

(a) Support on top layer

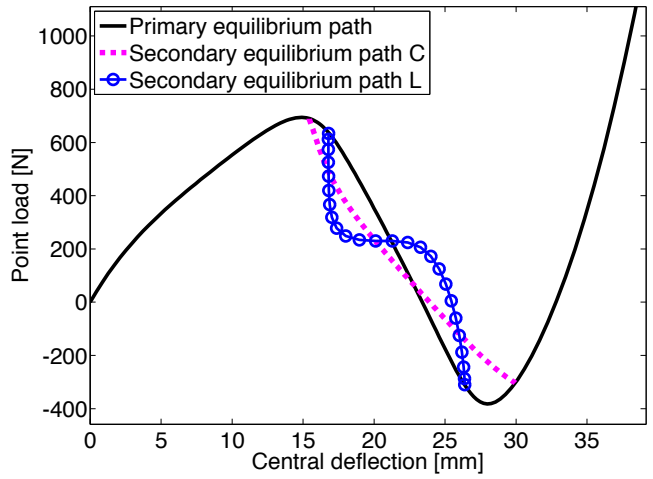

(b) Support on bottom layer

Figure 14: Primary and secondary paths of supports on two different layers

cedure combining the arclength with branch-switching methods is used to identify all critical points and trace all postbuckling responses. This numerical procedure has two major advantages over the one recommended in [36, 37]: (1) no prior knowledge of the bifurcation modes is required, and (2) the same mesh can be used to compute all secondary paths. Using this approach, two additional pairs of bifurcation points and secondary equilibrium paths are captured for the benchmark example. A small portion of the equilibria on one pair of secondary paths are stable, while all other equilibrium states on the bifurcated paths are unstable. These results provide a deeper understanding of the complex buckling and postbuckling behavior of the cylindrical panel and also contributes to the understanding of how the transient trajectories organize. 
An investigation of the influence of the rise, thickness and boundary conditions on the buckling and postbuckling behavior was also performed. It is found that these features have a great impact on the number and location of critical points and the postbuckling responses. Increasing the rise of the panel has the same effect on the appearance of bifurcation and limit-point buckling as decreasing the thickness. Multiple pairs of bifurcation points and secondary paths exist for cylindrical panels with a wide range of geometric parameters. Even when the same constraint is applied to the nodes on different layers in the thickness direction, the limit loads on the primary path, and the number and location of secondary paths are dramatically different.

\section{Acknowledgements}

The work has been funded by AFOSR under the grant no. FA9550121-1-0130 and by HPTI under Grant no. BY11020SP, BY12020SP, and BY13020SP. These supports are greatly appreciated. Support was also received from the Data Analysis and Visualization Cyber-infrastructure funded by NSF under grant OCI-0959097. We thank the reviewers for providing comments that helped us improve this manuscript 


\section{References}

[1] S. B. Batdorf, A simplified method of elastic-stability analysis for thin cylindrical shells. 2-Modified equilibrium equation (1947).

[2] L. H. Sobel, Effects of boundary conditions on the stability of cylinders subject to lateral and axial pressures, AIAA Journal 2 (1964) 1437-1440.

[3] N. J. Hoff, T. C. Soong, Buckling of circular cylindrical shells in axial compression, International Journal of Mechanical Sciences 7 (1965) 489520.

[4] L. H. Sobel, T. Weller, B. L. Agarwal, Buckling of cylindrical panels under axial compression, Computers and Structures 6 (1976) 29-35.

[5] G. Fischer, Influence of boundary conditions on stability of thin-walled cylindrical shells under axial load and internal pressure, AIAA Journal 3 (1965) 736-738.

[6] B. O. Almroth, Influence of edge conditions on the stability of axially compressed cylindrical shells., AIAA Journal 4 (1966) 134-140.

[7] N. Yamaki, Influence of prebuckling deformations on the buckling of 
circular cylindrical shells under external pressure., AIAA Journal 7 (1969) 753-755.

[8] D. O. Brush, Prebuckling rotations and cylinder buckling analysis, ASCE J Eng Mech Div 106 (1980) 225-232.

[9] K. Y. Volokh, O. Vilnay, On the prediction of geometrical non-linearity of slender structures, Communications in numerical methods in engineering 17 (2001) 19-29.

[10] M. Jabareen, Rigorous buckling of laminated cylindrical shells, ThinWalled Structures 47 (2009) 233-240.

[11] W. T. Koiter, The stability of elastic equilibrium, Technical Report AFFDL-TR-70-25, 1970.

[12] J. W. Hutchinson, W. T. Koiter, Postbuckling theory, Appl. Mech. Rev 23 (1970) 1353-1366.

[13] E. G. Carnoy, Asymptotic study of the elastic postbuckling behavior of structures by the finite element method, Computer Methods in Applied Mechanics and Engineering 29 (1981) 147-173. 
[14] R. Casciaro, G. Garcea, G. Attanasio, F. Giordano, Perturbation approach to elastic post-buckling analysis, Computers and Structures 66 (1998) 585-595.

[15] G. Garcea, A. Madeo, G. Zagari, R. Casciaro, Asymptotic post-buckling FEM analysis using corotational formulation, International Journal of Solids and Structures 46 (2009) 377-397.

[16] N. Damil, M. Potier-Ferry, A new method to compute perturbed bifurcations: application to the buckling of imperfect elastic structures, International Journal of Engineering Science 28 (1990) 943-957.

[17] B. Cochelin, N. Damil, M. Potier-Ferry, Asymptotic-numerical methods and pade approximants for non-linear elastic structures, International journal for numerical methods in engineering 37 (1994) 1187-1213.

[18] P. Vannucci, B. Cochelin, N. Damil, M. Potier-Ferry, An asymptoticnumerical method to compute bifurcating branches, International journal for numerical methods in engineering 41 (1998) 1365-1389.

[19] E. Boutyour, H. Zahrouni, M. Potier-Ferry, M. Boudi, Asymptoticnumerical method for buckling analysis of shell structures with large 
rotations, Journal of computational and applied mathematics 168 (2004) 77-85.

[20] A. Maewal, W. Nachbar, Stable postbuckling equilibria of axially compressed, elastic circular cylindrical shells: a finite-element analysis and comparison with experiments., Journal of Applied Mechanics, Transactions ASME 44 Ser E (1977) 475-481.

[21] J. L. Batoz, G. Dhatt, Incremental displacement algorithms for nonlinear problems, International Journal for Numerical Methods in Engineering 14 (1979) 1262-1267.

[22] E. Riks, An incremental approach to the solution of snapping and buckling problems, International Journal of Solids and Structures 15 (1979) $529-551$.

[23] M. Crisfield, A fast incremental/iterative solution procedure that handles "snap-through", Computers and Structures 13 (1981) 55-62.

[24] C. T. Tsai, A. N. Palazotto, Nonlinear and multiple snapping responses of cylindrical panels comparing displacement control and riks method, Computers and Structures 41 (1991) 605-610. 
[25] Sabir, AB, A. C. Lock, The applications of finite elements to large deflection geometrically nonlinear behaviour of cylindrical shells (1972).

[26] G. Horrigmoe, P. Bergan, Nonlinear analysis of free-form shells by flat finite elements, Computer Methods in Applied Mechanics and Engineering 16 (1978) 11-35.

[27] T. Hughes, W. K. Liu, Nonlinear finite element analysis of shells: Part I. three-dimensional shells, Computer Methods in Applied Mechanics and Engineering 26 (1981) 331-362.

[28] K. S. Surana, Geometrically nonlinear formulation for the curved shell elements., International Journal for Numerical Methods in Engineering 19 (1983) 581-615.

[29] S. Saigal, R. K. Kapania, T. Y. Yang, Geometrically nonlinear finite element analysis of imperfect laminated shells, Journal of Composite Materials 20 (1986) 197-214.

[30] C. H. Yeom, S. W. Lee, Assumed strain finite element model for large deflection composite shells, International Journal for Numerical Methods in Engineering 28 (1989) 1749-1768. 
[31] C. Sansour, H. Bufler, Exact finite rotation shell theory, its mixed variational formulation and its finite element implementation, International Journal for Numerical Methods in Engineering 34 (1992) 73-115.

[32] F. G. Flores, E. Oñate, F. Zarate, New assumed strain triangles for non linear shell analysis, Computational Mechanics. Solids, Fluids, Engineered Materials, Aging Infrastructure, Molecular Dynamics, Heat Transfer, Manufacturing Processes, Optimization, Fracture \& Integrity 17 (1995) 107-114.

[33] K. Y. Sze, S. J. Zheng, Hybrid stress nine-node degenerated shell element for geometric nonlinear analysis, Computational Mechanics. Solids, Fluids, Engineered Materials, Aging Infrastructure, Molecular Dynamics, Heat Transfer, Manufacturing Processes, Optimization, Fracture \& Integrity 23 (1999) 448-456.

[34] K. Y. Sze, W. K. Chan, T. H. H. Pian, An eight-node hybrid-stress solid-shell element for geometric non-linear analysis of elastic shells, International Journal for Numerical Methods in Engineering 55 (2002) 853-878.

[35] K. Y. Sze, X. H. Liu, S. H. Lo, Popular benchmark problems for ge- 
ometric nonlinear analysis of shells, Finite Elements in Analysis and Design 40 (2004) 1551-1569.

[36] B. Wardle, P. A. Lagace, M. A. Tudela, Buckling response of transversly loaded composites shells, Part 2: Numerical analysis, AIAA Journal 42 (2004) 1465-1473.

[37] B. L. Wardle, Solution to the incorrect benchmark shell-buckling problem, AIAA Journal 46 (2008) 381-387.

[38] W. Wagner, P. Wriggers, Simple method for the calculation of postcritical branches., Engineering Computations (Swansea, Wales) 5 (1988) 103-109.

[39] P. Wriggers, J. Simo, General procedure for the direct computation of turning and bifurcation points, International Journal for Numerical Methods in Engineering 30 (1990) 155-176.

[40] R. L. Taylor, FEAP- Finite Element Analysis Program, University of California, Berkeley, 2014. URL: http://www.ce.berkeley.edu/ projects/feap/.

[41] R. Wiebe, I. Stanciulescu, Inconsistent stability of newmarks method in 
structural dynamics applications, Journal of Computational and Nonlinear Dynamics (2014). 